\title{
A new approach to treating chronic infections
}

\author{
Alexander P. Malyshkin \\ Correspondence: malishkin_54@mail.ru \\ Orenburg State Medical Academy, Orenburg, Russia.
}

\begin{abstract}
There is strong evidence against the common view on a chronic infection as a consequence of the pathogen's antibiotic resistance and/or host's immune system insufficiency. A different explanation is offered by the active susceptibility concept. It postulates that a macroorganism is only susceptible to infection by the microorganisms, pathogenic and nonpathogenic alike, that produce certain substances required for the host's normal metabolism. This usually results in the formation of normal microflora, or "healthy carrier" cases, with an infectious disease as an occasional side effect. The possible practical implication is that administration of the products of the corresponding bacterial/viral gene to the host is expected to "switch off" the active susceptibility to the given infection, thus preventing or curing it. In the case of a plant or animal infectious disease, the gene can be directly integrated into the host's genome by genetic engineering methods.
\end{abstract}

Keywords: Chronic infection, prevention, treatment, normal microflora

\section{To the Editor}

The beginning of the antibiotic era gave physicians a strong hope for eradication of chronic infectious diseases. Nevertheless, chronic infections still flourish, and the details of their formation, as well as the possible ways of their treatment, remains to be understood. The antibiotic resistance of the microflora and defects of the immune system are hypothesized to underlie the difficulties with the treatment of chronic infections. It is known, however, that in vivo antibacterial therapy of these diseases often proves ineffective even if their pathogens are sensitive to antibiotics in vitro; regarding the immunity, its parameters are unaltered in many cases of such infections. At the same time, recent concepts about the susceptibility to contagious infections suggest a different approach to treating these diseases. The active susceptibility concept $[\mathbf{1 , 2}]$ emphasizes the absence of fundamental differences between the normal and pathogenic infective microfloras. Both are infective, and both may either cause diseases or be useful for the host in the state of healthy carriage. According to this concept, the susceptibility to the normal microflora is (due to its necessity) an active property of the host microorganism. Hence, it is conceivable that the susceptibility to the pathogenic microflora is also active, and this is why pathogens are infective for the host. This means that microorganisms do not "force themselves" on the host; instead, the host body actively infects itself with the microorganisms that are necessary for it. In other words, by analogy with chronic foci of normal microflora, all other foci of chronic infection are also formed by the host body rather than microorganisms, because these foci are required for the body's normal functions. It remains to be understood what these functions are. However, many pathogenic bacteria are already known to be useful in the cases of healthy or asymptomatic carriage. Furthermore, the hygiene hypothesis relates the current increase in noninfectious somatic (e.g., allergic and endocrine) morbidity with excessive antimicrobial hygienic measures. The active susceptibility concept, which may be regarded as the theoretical substantiation of the hygiene hypothesis, assumes that the susceptibility to an infective microorganism results from the necessity of the products of some of its genes for the host body. Therefore, incorporation of certain microbial genes into the genome of a susceptible host should lead to the elimination of the susceptibility to the corresponding pathogenic organism. An application for the invention of this method for preventing infectious diseases has been published [3]. It is noteworthy that this invention can be used not only for preventing infectious diseases (and breeding animals and plants resistant to certain infections), but also for treating a chronic animal and human infections, including HIV/AIDS. I would be grateful to all researchers who wish to collaborate with me in developing these preventive and therapeutic methods.

\section{Competing interests}

The author declares no competing interest.

Acknowledgement

I thank V.L. Ushakov for the assistance in preparing the manuscript.

Publication history

Received: 13-Mar-2013 Accepted: 02-Apr-2013

Published: 20-Apr-2013

\section{References}

1. Malyshkin AP: Infection: a hypothesis on active susceptibility and species immunity with implications for AIDS prevention. Immunobiology 2010, 215:894-7. | Article | PubMed 
Alexander P. Malyshkin Research Journal of Infectious Diseases 2013,

2. Malyshkin AP: Prospects of prevention of infectious diseases in plants, animals, and humans. In: Sridar KR (Ed.). Aquatic Plants and Plant Diseases. New York: Nova Science Publishers 2012, 173-192. | Book

3. Malyshkin AP: Method for preventing infectious diseases of plants, animals and humans. WO/2011/084090. | Website

\section{Citation:}

Malyshkin AP: A new approach to treating chronic

infections. Research Journal of Infectious Diseases 2013, 1:1. http://dx.doi.org/10.7243/2052-5958-1-1 